\title{
Pollen success, functional gender and assortative mating in an experimental plant population
}

\author{
R. A. Ennos and \\ R. K. Dodson
}

\begin{abstract}
Department of Forestry and Natural Resources, University of Edinburgh, The King's Buildings, Mayfield Road, Edinburgh EH9 3JU.
\end{abstract}

Six uniquely genetically marked individuals were extracted from a natural population of the perennial, selfincompatible grass Cynosurus cristatus. Individuals were clonally replicated and used to construct an experimental population within which pollination and seed set were allowed to occur. The relative allocation to female reproduction, and the pollen contribution to progeny were measured for each clone. A model was developed to predict the expected pollen contribution of each clone on the assumption that clones showed a constant ratio of male/female reproductive resource allocation and equal pollen fitness. Highly significant differences were found between observed and expected pollen contributions of clones. Two clones showed significant deviations from equisexual reproduction. Differences in flowering time among clones led to assortative mating between clones which flowered synchronously, and this was accompanied by changes in the genotypic composition of the pollen pool through the flowering season.

\section{INTRODUCTION}

Genetic transmission in hermaphrodite plant populations has been studied, almost exclusively, within the conceptual framework of the mixed mating model (Fyfe and Bailey, 1951; Hayman, 1953). The model comprises two components; a population of maternal parents, and an outcross pollen pool. Mating occurs when maternal parents either self-fertilise, or outcross by receiving pollen randomly from the outcross pollen pool. Though this model has proved invaluable in the quantitative analysis of plant mating systems (Clegg, 1980), it provides an incomplete description of the mating process. It fails to take account of the link between the genotypic composition of the parental population and that of the pollen pool. Attention is concentrated on the functioning of hermaphrodite plants as maternal parents, neglecting their role as pollen donors (Horovitz and Harding, 1972).

The deficiencies of the model have been brought into focus by recent attempts to measure the functional gender of cosexual plants, their relative reproduction as male and female parents (Lloyd, 1980; Primack and Lloyd, 1980). It has generally been assumed that hermaphrodite plants behave in an equisexual manner, i.e. each individual contributes equally to the next gener- ation as a male and as a female parent. However, there is very little genetic evidence to support such an assumption, for few attempts have been made simultaneously to follow the genetic contributions of individuals as male and female parents to the next generation (Horovitz, 1978; Lloyd, 1980; Ross and Gregorius, 1983). Indeed measurement of male and female genetic contributions in seed orchards of the monoecious Pinus sylvestris suggests that large deviations from equisexual reproduction may be common (Muller-Starck and Ziehe, 1984). The work outlined in this paper is an attempt to determine whether similar deviations from equisexual reproduction are likely to occur within populations of the hermaphrodite selfincompatible grass Cynosurus cristatus.

The functional gender of an individual within a population is dependent upon three factors:

(a) relative resource allocation to male and female reproduction (pollen grains/seed weight).

(b) probability that pollen produced takes part in. fertilisation of successful zygotes.

(c) probability that seeds produced are included in the next generation.

In practice it is extremely difficult to measure both pollen and seed success. Estimates of "prospective gender" have therefore been made in which constant pollen and seed success over genotypes 
has been assumed. Prospective gender is predominantly male if the ratio of male/female resource allocation exceeds the population mean, whereas it is predominantly female when this ratio falls below the population mean (Lloyd, 1980; Primack and Lloyd, 1980). Further refinements of this estimation technique can be applied to take account of variation in the success of pollen through the flowering season (Thomson and Barrett, 1981) or with respect to plant position within the population.

A closer approximation to a true estimate of functional gender can be obtained, however, if the success of an individual's pollen can be measured directly, so that assumptions about the fate of pollen need no longer be made. This objective can be achieved if the pollen of each plant in a population is uniquely genetically marked, and its inclusion in the progeny generation can be readily detected.

With this in mind, a population was constructed in which pollen success could be monitored using genetic markers, while at the same time the female resource allocation of each individual could be measured. A model was then developed which assumed that all genotypes showed a constant ratio of male to female resource allocation, and equal probability of success of their pollen grains. The observed pollen contributions of genotypes were then compared with those predicted by the model to determine whether significant deviations from equisexual reproduction had occurred.

\section{MATERIALS AND METHODS}

C. cristatus is a perennial, self-incompatible, windpollinated grass which is a common component of permanent pastures in Britain. Populations are polymorphic at four diallelic loci, MDH1, MDH2, $\mathrm{ADH}$ and GOT, each of which shows regular Mendelian inheritance (Ennos, 1986). In the course of an investigation of genetic differentiation within a natural population (Ennos, 1985), six different genotypes homozygous at these four loci were identified (table 1). The results of controlled crossand self-pollination have demonstrated that the six genotypes are highly self-incompatible, but interfertile.

Once identified, the six genotypes were vegetatively propagated by rooting tillers from the parent plants in $10 \mathrm{~cm}$ pots. Sixteen replicates of each clone were grown for 6 months in the greenhouse in a completely randomised block. In August 1983 the 96 individuals ( 6 clones $\times 16$ replicates) were
Table 1 Homozygous genotypes of clones used in the experimental population: $\mathrm{F}=$ fast migrating allele, $\mathrm{S}=$ slow migrating allele

\begin{tabular}{lllll} 
Clone & Mdh-1 & Mdh-2 & Adh & Got \\
\hline 1 & F & S & F & S \\
2 & F & F & S & S \\
3 & F & F & F & F \\
4 & S & S & F & F \\
5 & F & S & F & F \\
6 & F & F & F & S \\
\hline
\end{tabular}

taken outside and their pots were sunk in a sand bed which was well isolated from any natural populations of $C$. cristatus. Replicates were arranged in an $8 \times 12$ array at a spacing of $20 \mathrm{~cm}$ in a completely randomised block. After vernalisation, flowering occurred during June and July 1984.

For each clone the number of flower spikes releasing pollen was recorded through the flowering season. After seed set all flowering spikes from each replicate were harvested separately. The length of each flowering spike and the number of seeds set per spike were recorded. A random sample of 40 seeds was weighed from each replicate of the six clones. These data were used to estimate reproductive performance of clones as female parents.

In order to measure the pollen contribution of each clone to the progeny, a sample of one twentieth of the seed set from each spike was grown up by maternal parent in seed trays. A fixed proportion rather than a fixed number of seed was grown from each spike in order to obtain an unbiased estimate of pollen success within the complete population. Each of these progeny was scored for its genotype at the four isozyme loci. From a knowledge of the zygotic and maternal genotype of each individual, the paternal genotype could be inferred.

\section{RESULTS AND ANALYSIS}

\section{Resource allocation to female reproduction}

Table 2 gives the total number and length of flower spikes, total seed numbers, and estimated total seed weight of the six clones in the experimental population. There is a threefold difference among clones in the absolute resource allocation to female reproduction (measured as total seed weight). Female resource allocation is not dependent upon the size of the fixed investment in reproduction (measured as length of flower spikes) since analysis 
Table 2 Summary of data collected on reproduction of clones as female parents. The significance of differences among clones for the characters seed weight $/ \mathrm{mm}$ spike, seed number/mm spike and mean seed weight were tested using a nested analysis of variance. ${ }^{* * *} P<0.001$

\begin{tabular}{|c|c|c|c|c|c|c|c|}
\hline & Clone 1 & Clone 2 & Clone 3 & Clone 4 & Clone 5 & Clone 6 & $F_{5,90}$ \\
\hline Total spike number & 191 & 153 & 51 & 147 & 132 & 107 & \\
\hline Total spike length (mm) & 5740 & 4905 & 1509 & 5083 & 4916 & 3737 & \\
\hline Total seed number & 6751 & 7839 & 2097 & 5591 & 8746 & 5723 & \\
\hline Total seed weight (gms) & $3 \cdot 204$ & $5 \cdot 940$ & $1 \cdot 609$ & $4 \cdot 796$ & $5 \cdot 316$ & $2 \cdot 978$ & \\
\hline Seed weight/mm spike (mg) & 0.559 & $1 \cdot 211$ & 1.066 & 0.944 & 1.082 & 0.796 & $8.50^{* * *}$ \\
\hline Seed number/mm spike & $1 \cdot 145$ & 1.598 & $1 \cdot 390$ & $1 \cdot 100$ & 1.779 & $1 \cdot 531$ & $6 \cdot 69^{* * *}$ \\
\hline Mean seed weight (mg) & 0.488 & 0.758 & 0.767 & 0.858 & 0.608 & 0.520 & $47 \cdot 84^{* * *}$ \\
\hline
\end{tabular}

of variance shows highiy significant differences in weight of seed per unit of spike length $(P<0.001$ table 2).

The differences in seed weight per unit of spike length are brought about not only by significant variation in seed number per unit of spike, but also by significant variation in mean seed weight among clones $(P<0.001$ in both cases $)$. This marked variability in the resources devoted to each zygote by different clones means that seed number may not be a reliable indicator of the likely rate of transmission of gametes via ovules to the next generation. Gametes include in progeny of clones producing heavier seeds are far more likely to contribute to the next generation than are those from clones producing lighter seeds (Black, 1958; Rhodes, 1968; Cideciyan and Malloch, 1982).

\section{Contribution of clones as male parent}

Table 3 gives the paternity and maternity of the progeny classified by electrophoretic analysis. The table excludes individuals produced by self-pollination $(0.3$ per cent of total) and by pollen from outside the experimental population $(0.4$ per cent of total). The question of interest is whether such a progeny array is expected on the assumption that all clones show a constant ratio of male/female resource allocation and equal pollen fitness. In order to answer this we must construct a simple model of mating within the experimental population, taking into account the unusual incompatibility structure which it possesses. The aim of the model is to generate expected numbers of progeny genotypes for clones acting as male and female parents in all possible combinations. Expected numbers can then be compared with the observed values which appear in table 3 .

\section{Model assumptions}

(a) The population is composed of six clones which are self-incompatible but fully interfertile.

(b) The ratio (resource allocation to male reproduction (pollen grains))/(resource allocation to female reproduction (seed weight)) is constant over all clones.

(c) Seed production is not limited by pollen availability.

(d) Success of a clone as a pollen parent is proportional to the frequency of its pollen in the eligible pollen pool (total pollen less the pollen produced by the clone being fertilised), i.e. there are no differences in the fitness of pollen types.

(e) Pollen is randomly distributed over eligible clones.

Table 3 Maternity and paternity of progeny analysed by electrophoresis

\begin{tabular}{|c|c|c|c|c|c|c|c|c|}
\hline & & \multicolumn{6}{|c|}{ Female parent } & \multirow[b]{2}{*}{ Total } \\
\hline & & Clone 1 & Clone 2 & Clone 3 & Clone 4 & Clone 5 & Clone 6 & \\
\hline \multirow{4}{*}{ Male parent } & Clone 1 & - & 36 & 4 & 41 & 90 & 69 & 240 \\
\hline & Clone 3 & 20 & 47 & - & 23 & 48 & 24 & 162 \\
\hline & Clone 4 & 81 & 98 & 24 & - & 130 & 77 & 410 \\
\hline & Clone 5 & 84 & 161 & 44 & 111 & - & 58 & 458 \\
\hline
\end{tabular}




\section{Model}

We define the following parameters for $i=1, \ldots, 6$

$m_{i}=$ pollen production by clone $i$,

$f_{i}=$ total seed weight of clone $i$,

$n_{i}=$ number of seeds of clone $i$ classified by electrophoresis.

We note from (b) above that $m_{i} / f_{i}=K=$ constant Then in order to calculate the expected number of progeny with clone $\underline{a}$ as female parent, and clone $b$ as male parent:

pollen production by clone $\underline{b}=m_{b}=K f_{b}$, and total pollen eligible to fertilise clone $\underline{a}=$ $\sum_{i=1}^{6} m_{i}-m_{a}=K\left(\sum f_{i}-f_{a}\right)$.

Frequency of pollen $\underline{b}$ in eligible pollen pool of clone $\underline{a}$ equals

$$
\frac{K f_{b}}{K\left(\sum f_{i}-f_{a}\right)}=\frac{f_{b}}{\sum f_{i}-f_{a}}
$$

Expected number of progeny with clone $\underline{a}$ as female parent, and clone $b$ as male parent equals

$$
\frac{n_{a} f_{b}}{\sum f_{i}-f_{a}} .
$$

By substituting the values for total seed weight of each clone $\left(f_{i}\right)$, and number of seeds of each clone scored by electrophoresis $\left(n_{i}\right)$ into this formula the expected numbers of progeny in each cell of table 3 were calculated. The observed and expected pollen contributions of each clone to each maternal parent are illustrated for comparison in fig. 1. Also illustrated are the observed and expected total pollen contributions of each clone. A $\chi^{2}$ statistic can be used to test the goodness of fit of the model. There are 30 observations and 6 constraints leaving 24 degrees of freedom. The model gives an extremely poor fit to the data $\left(\chi_{(24)}^{2}=\right.$ 197.95, $P<0.001)$. The discrepancies between observed and expected pollen contributions of clones can be discussed under two headings; deviations from expected total contribution as male parent, and deviation from expected pollen distribution over maternal clones.

For clones 1, 3, 4 and 6 the observed total pollen contributions are very close to those expected under the assumptions of the model. In contrast, clone 2 contributes far less pollen, and clone 5 far more pollen to progeny than expected. In the experimental population clone 2 shows female predominant reproduction, whereas clone 5 shows male predominant reproduction. The deviations from expected pollen success of the clones could have arisen for a number of reasons. In the first place they could be due to deviations from the mean male/female reproductive resource allocation of clones, so that clone 2 devoted less than and clone 5 more than the average proportion of their reproductive resources to pollen. An alternative explanation is that the male/female resource allocations are equal to the population mean, but the fitness of clone 2 pollen is lower, and the fitness of clone 5 pollen is higher than the mean population pollen fitness. Unfortunately we cannot distinguish between the differential resource allocation and the differential pollen fitness hypotheses. Nevertheless, these results show that within the natural population deviations from equisexual reproduction of clones are likely to occur, so that clone 2 will tend to transfer a higher proportion of its gametes via ovules than via pollen, whereas clone 5 will transfer more male gametes to progeny than female gametes.

Turning to the distribution of pollen over maternal genotypes, we can see that this shows highly significant deviations from the pattern expected under the model. Thus pollen of clone 1 fertilises ovules of clone 6 far more frequently than expected, but fertilises ovules of clones 2 and 3 far less frequently than expected. The differential fertilisation of certain maternal clones could arise for either of two reasons. Firstly the patterns of fertilisation could reflect differences in the degree of compatibility among clones. Unfortunately the details of the incompatibility system of $C$. cristatus have not been elucidated, though it is thought to possess a two locus gametophytic system similar to that of Lolium perenne (Weimarck, 1968, Cornish et al., 1979). The low frequency of fertilisation in particular combinations may be due to half or three-quarter compatibility between clones. In the absence of further information we cannot pursue this explanation.

An alternative explanation for the differential success of pollen genotypes over maternal parents is that this reflects patterns of assortative mating caused by differences in flowering item among clones. According to this explanation we should expect an excess of fertilisation events between clones of like flowering time, and a deficit of fertilisation events between clones of unlike flowering time. Fig. 2 gives data on pollen release by clones throughout the flowering season. Despite the absence of recordings in the middle of the season, it is clear from the figure that clones exhibit highly significant differences in date of pollen release. Clones 1 and 6 flower first, followed by clones 4 and 5 . Clones 2 and 3 are the last to flower. Given this sequence of flowering we expect positive assortative mating between the early flowering 


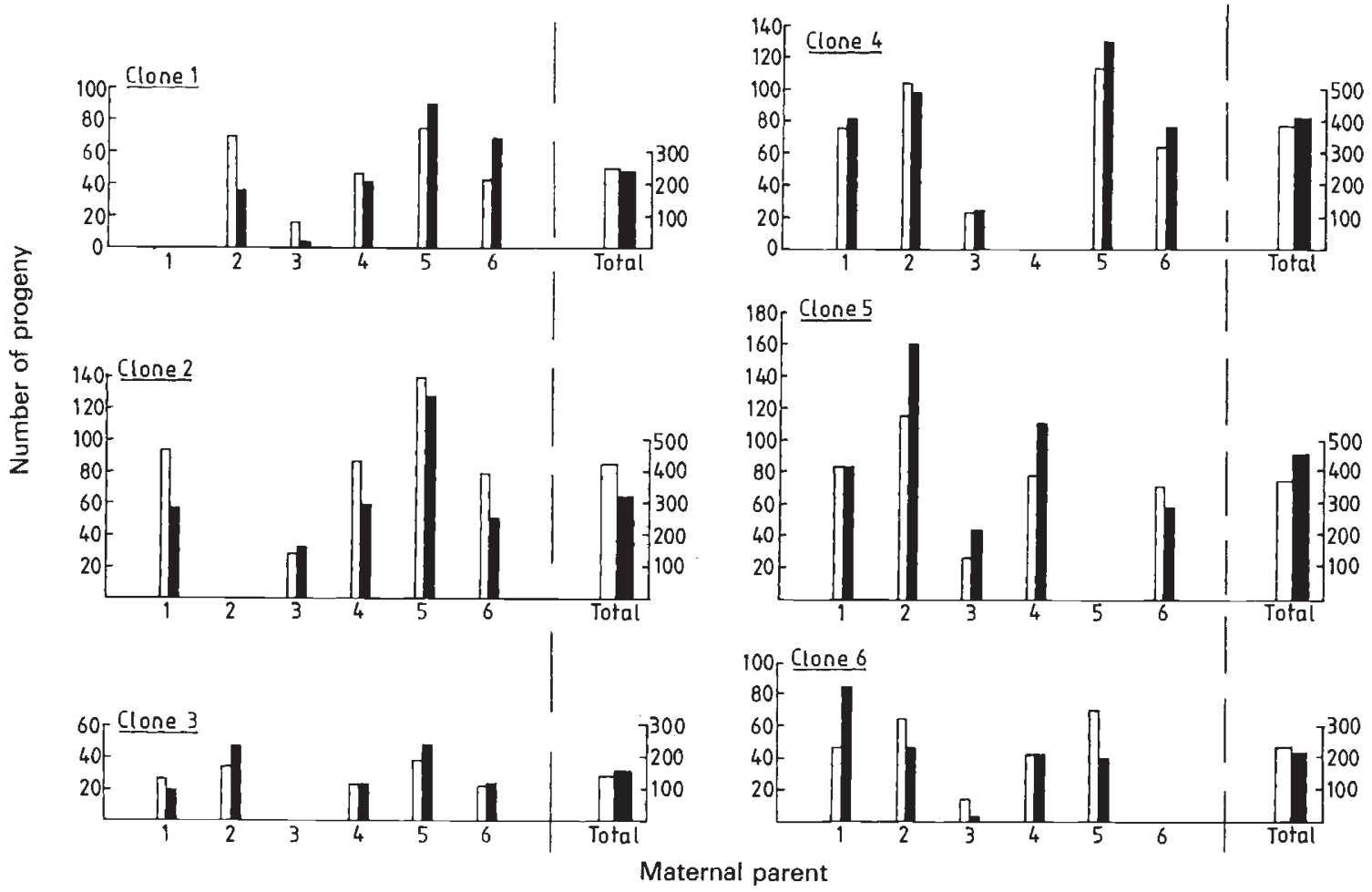

Figure 1 Comparison of observed (shaded bars) and expected (open bars) pollen contributions of each clone to maternal parents. Observed and expected total pollen contributions of each clone are shown to the right of the figure.

clones (1 and 6) and between the late flowering clones ( 2 and 3 ). Conversely we expect a deficit of matings between these early and late flowering groups. Inspection of Fig. 1 bears out these predictions, suggesting that assortative mating with respect to flowering time is a major determinant of mating pattern within the experimental population.

Further evidence for the existence of assortative mating can be obtained by monitoring the genotype frequencies present in the pollen pool at different times through the flowering season. If flowering time has a significant effect on mating pattern we expect to be able to detect changes in pollen genotype frequencies over time, changes which are related to the flowering sequence of the six clones.

In order to investigate this possibility, 5 seeds were taken from the earliest flowering spike of each replicate of clone 1 , and 5 seeds from the latest flowering spike of each replicate. These progeny were grown separately and scored for their genotype at the marker loci. From these data the frequency of pollen from each clone encountered by the early and late flowering spikes of clone 1 was inferred. A similar procedure was carried out for clone 2 . The results are presented in table 4 .

With clone 1 as female parent, a significant increase in the frequency of pollen from clone 2 occurs over the season $\left(\chi_{(1)}^{2}=8.62, P<0.01\right)$, accompanied by a significant decrease in the frequency of pollen of clone $6\left(\chi_{(1)}^{2}=6.64, P<\right.$ $0 \cdot 01)$. This is fully compatible with the evidence already presented that clone 2 flowers later, whereas clone 6 flowers synchronously with clone 1. For clone 2 as female parent, a significant decrease in the frequency of pollen from clone 5 is found over the flowering period $\left(\chi_{(1)}^{2}=6 \cdot 35\right.$, $P<0 \cdot 01)$. Again this is entirely consistent with the observation that clone 5 flowers earlier than clone 2.

These analyses of changes in the genotypic composition of the pollen pool through the flowering season provide additional support for the hypothesis that assortative mating by flowering time is taking place within the experimental population, and is a major determinant of interclonal mating patterns. 


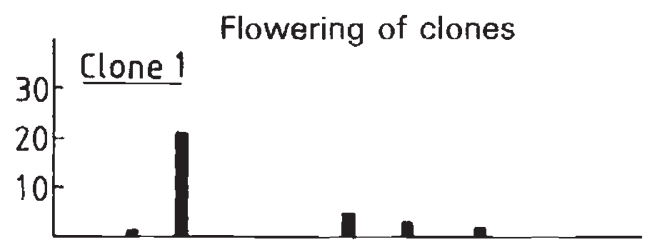

40 Clone 2
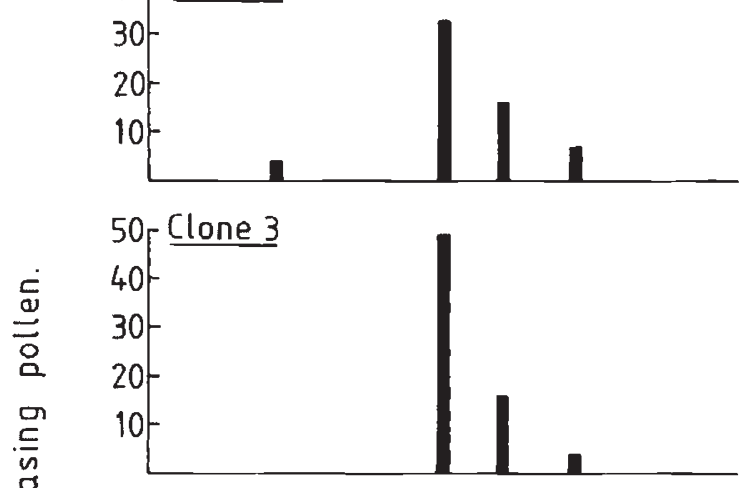

(

品

30 Clone 4

20
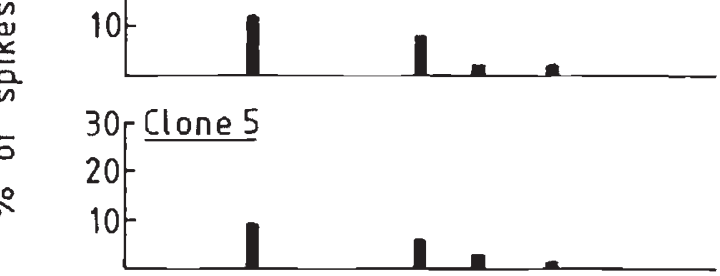

30 Clone 6

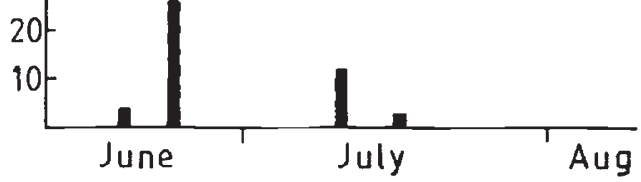

Figure 2 Percentage of flower spikes of each clone releasing pollen at various times through the flowering season.

\section{DISCUSSION}

There has been much debate about the potential for hermaphrodite plants to show variation in functional gender, the relative proportion of genes transmitted to the next generation via pollen and ovules (Lloyd, 1980). Horovitz (1978) and Ross and Gregorius (1983) have proposed that variation in functional gender may be a common phenomenon within hermaphrodite populations whose members have traditionally been assumed to function equally as male and female parents. It has also been suggested that this variation may be under a certain amount of genetic control.
Experimental evidence on these topics has been provided by Primack and Lloyd (1980) and Thomson and Barrett (1981) who demonstrated large variation in prospective gender within a total of three natural plant populations. Large deviations from equal functioning of clones as male and female parents have also been shown in seed orchards of monoecious conifers (Eriksson et al., 1973; Jonsson et al., 1976; Muller-Starck and Ziehe, 1984). Unfortunately, the seed orchards used in these studies were composed of clones from a variety of provenances, and much of the variation in functional gender could be ascribed to the variability in male and female flowering behaviour between provenances. A major conclusion from these studies was that wide fluctuations in the functional gender of individuals may occur from year to year, suggesting that the trait is likely to have a very low heritability (Lloyd and Bawa, 1984).

In the present experiment the six clones used were all collected from a single field and any differences in flowering behaviour among clones should represent the range of variation present within a natural population. Two of the six clones comprising the experimental population showed pollen contributions which differed widely from those expected under the assumptions of constant ratio of resource allocation to male and female reproduction, and equal fitness of pollen from each clone. This provides further evidence that deviations from equisexuality may be common in hermaphrodite plant populations. It should always be borne in mind, however, that the functional gender shown by an individual is critically dependent upon the composition and flowering behaviour of the population in which it is measured. The large deviations from expected pollen contributions recorded here may be caused by a restricted choice of maternal genotypes available for pollen to fertilise. Indeed, one possible explanation for the low pollen success of clone 2 may be related not to low resource allocation to male reproduction, but rather to a lack of eligible receptive stigmas and unfertilised ovules late in the season when the bulk of pollen was released from clone 2. This effect has been noted previously by Thomson and Barrett (1981) who demonstrated the importance of variation in pollen/ovule ratios from day to day in determining the functional gender of Aralia hispida. Further work is required on the male to female resource allocation strategies and the compatibility of clones before the relative importance of flowering time effects on functional gender can be assessed in this experimental population of $C$. cristatus. 
Table 4 Comparison of pollen contributions by the six clones in the production of progeny on early and late flowering spikes of clone 1 and 2 . Significant differences in the frequency of pollen types between early and late samples are indicated. ${ }^{*} P<0.01$

\begin{tabular}{|c|c|c|c|c|c|c|c|c|}
\hline & & \multicolumn{6}{|c|}{ Pollen from } & \multirow[b]{2}{*}{ Total } \\
\hline & & Clone 1 & Clone 2 & Clone 3 & Clone 4 & Clone 5 & Clone 6 & \\
\hline \multirow{3}{*}{ Female parent } & Clone 1 early & - & 4 & 2 & 18 & 20 & 35 & 79 \\
\hline & Clone 2 early & 14 & - & 7 & 11 & 41 & 4 & 77 \\
\hline & Clone 2 late & 14 & - & 14 & 18 & $25^{*}$ & 6 & 77 \\
\hline
\end{tabular}

The investigation described here uses an indirect method for assessing functional gender. Ross and Gregorius (1983) have proposed an alternative, direct measure of functional gender based on counts of the relative numbers of "successful" gametes transferred to zygotes via ovules and pollen. "Successful" gametes are defined as gametes, either male or female, which take part in the formation of zygotes. Under the model of Ross and Gregorius all zygotes receive equal maternal investments, and therefore have equal probabilities of contributing to the next generation. Under these conditions simple counts of numbers of male and female gametes contributing to seed provide an unbiased and direct estimate of functional gender.

The situation in the present experiment is that highly significant differences in individual seed weight were found among clones $(P<0.001$, table 2 ). The zygotes comprising the progeny population are unlikely to have equal probabilities of contributing to the next generation. Those receiving a large maternal investment may have a higher probability of contributing than those receiving low maternal investment (Black, 1958; Rhodes, 1968; Cideciyon and Malloch, 1982). Thus the relative contributions of clones as male and female parents cannot be measured simply by counting frequencies of gametes contributed via pollen and ovules to the seed population. If we wished to make a direct measure of "successful" gametes in this situation, the frequency of male or female gametes contributed would have to be weighted by the relative maternal fitness of the seeds in which they were included.

Given that deviations from equisexuality are likely to occur in hermaphrodite populations, we can consider the implications which this has for studies of natural selection. Firstly, $i \hat{i}$ should make us cautious in using estimates of fitness based solely on measures of seed production. If functional gender is male biased, fitness will be underestimated, whereas if it is female biased we will overestimate fitness. The second point to note is that functional gender affects the dispersal distance of gametes from the parent plant (Lloyd, 1982). Plants biased towards male reproduction will dis- perse their gametes farther than those biased towards female reproduction. This has implications for the measurement of neighbourhood size, the degree of competition between related progeny, and the advantages of combining or separating male and female functions (Lloyd, 1984).

Apart from the information provided about functional gender, this experiment has also revealed highly significant assortative mating by flowering time within the experimental population. Despite the fact that this effect is widely acknowledged to occur, there appears to be only one previous demonstration of the phenomenon using genetic markers (Gutierrez and Sprague, 1959). The paucity of data on this subject can partly be accounted for by the fact that a mixed mating model analysis is unlikely to detect assortative mating, since the origin of pollen taking part in fertilisation events cannot be traced. Indeed analysis of the breeding system in the natural population from which the present experimental material was extracted, led to the conclusion that mating took place by random outcrossing (Ennos, 1985). The present study reveals that outcrossing is likely to be highly non-random, with successful fertilisation being most likely between genotypes of similar flowering time. The data on changes in the genotypic composition of the pollen pool over time reveals another way in which the assumptions of the mixed mating model can be violated. It is already known that substructuring of the population can lead to non-random distribution of pollen over maternal genotypes (Ennos and Clegg, 1982). Here we show that temporal variation in the genetic composition of the pollen pool leads to nonrandom distribution of pollen over maternal genotypes.

Many processes interact to produce the final array of progeny genotypes in this experimental population. Variation in functional gender of clones and assortative mating among clones are two of the processes which have been identified. The analysis has been facilitated by our ability to follow the fate of genetically marked pollen from paternal parent to progeny. In the absence of information about paternity, application of mixed 
mating model analysis to the same data would merely have indicated significant differences in allele frequencies between the adult population and the pollen pool. We would have been left to speculate on how these could have arisen. The deeper understanding of the breeding system which has been gained by following the fate of genetically marked pollen is a strong argument for undertaking the extra work required for such a study.

The extension of paternity analysis from experimental to natural populations is an obvious next step in unravelling the complexities of plant mating systems (Ellstrand, 1984; Hamrick and Schnabel, 1985). The challenges presented are formidable, both in terms of finding sufficient genetic markers unambiguously to identify pollen from individual genotypes, and in terms of assaying a sufficient number of progeny from the population. There is probably more to be gained in the immediate future through the use of experimental populations in which the genotypic composition and degree of genetic isolation are under strict control.

Acknowledgements We would like to thank Dr D. G. Lloyd and an anonymous reviewer for their very helpful comments on the manuscript. John Morman provided invaluable technical assistance. This work was supported by a grant from the Nuffield Foundation to Richard A. Ennos.

\section{REFERENCES}

BLACK, J. N. 1958. Competition between plants of different initial seed sizes in swards of subterranean clover (Trifolium subterraneum $L$.) with particular reference to leaf area and the light microclimate. Aust. J. Agric. Res., 9, 299-318.

CIDECIYAN, M. A. AND MALLOCH, A. J. C. 1982. Effects of seed size on the germination, growth and competitive ability of Rumex crispus and Rumex obtusifolius. J. Ecol., 70, $227-232$

CleGG, M. T. 1980. Measuring plant mating systems. Bio Science, 30, 814-818.

CORNISH. M. A. HAYWARD, M. D., AND LAWRENCE, M. J. 1979. Self-incompatibility in ryegrass I. Genic control in diploid Lolium perenne L. Heredity, 43, 95-106.

ELLSTRAND, N. C. 1984. Multiple paternity within the fruits of the wild radish. Amer. Natur. 123, 819-828.

ENNOS, R. A. 1985. The mating systen and genetic structure in a perennial grass, Cynosurus cristatus L. Heredity, 55, 121126.
ENNOS, R. A. 1986. Allozyme variation, linkage and duplication in the perennial grass Cynosurus cristatus. J. Hered, 77, 61-62.

ENNOS, R. A. AND CLEGG, M. T. 1982. Effect of population substructuring on estimates of outcrossing rate in plant populations. Heredity, 48, 283-292.

ERIKSSON, G., JONSSON, A., AND LINDGREN, O. 1973. Flowering in a clone trial of Picea abies. Studia Forestalia Suecia No. 110

FYFE, J. I., AND BAILEY, N. T. J. 1951. Plant breeding studies in leguminous forage crops. 1. Natural cross-breeding in winter beans. J. Agric. Sci., 41, 371-378.

GUTIERREZ, M. G. AND SPRAGUE, G. F. 1959. Randomness of mating in isolated polycross plantings of maize. Genetics, 44, $1075-1082$.

HAMRICK, J. L., AND SCHNABEL, A. 1985. Understanding the genetic structure of plant populations: some old problems and a new approach. Gregorius, H-.R. (ed.). In Population Genetics and Forestry, Lecture Notes in Biomathematics 60, Springer-Verlag, Berlin.

HAYMAN, B. 1. 1953. Mixed selfing and random mating when homozygotes are at a disadvantage. Heredity, 7, 185-192.

HOROVITZ, A. 1978. Is the hermaphrodite flowering plant equisexual? Amer. $J l$. Bot., 65, 485-486.

HOROVITZ, A., ANI HARDING, J, 1972. The concept of male outcrossing in hermaphrodite higher plants. Heredity, 29 , 223-236

JONSSON, A., EKBERG, I. AND ERIKSSON, G, 1976. Flowering in a seed orchard of Pinus sylvestris. Studia Forestalia Suecia No. 135 .

LLOYD, D. G. 1980. Sexual strategies in Plants. III. A quantitative method for describing the gender of plants. N.Z. $J$. Bot., 18, 103-108.

L.LOYD, D. G. 1982. Selection of combined versus separate sexes in seed plants. Amer. Natur., 120, 571-585.

LLOYD, D. G. 1984. Variation strategies of plants in heterogeneous environments. Biol. J. Linn. Soc., 21, 357. 385 .

LLOYD, D. G. AND BAWA, K. S. 1984. Modification of the gender of seed plants in varying conditions. Evol. Biol., 17, 225 338.

MULLER-STARCK, G. AND ZIEHE, M. 1984. Reproductive systems in conifer seed orchards. 3. Female and male fitnesses of individual clones realised in seeds of Pinus sylvestris $\mathrm{L}$. Theor. Appl. Genet., 69, 173-177.

PRIMACK, R. B., AND ILOYD, D. G. 1980. Sexual strategies in plants. IV. The distribution of gender in two monomorphic shrub populations. N.Z. Jl. Bot., 18, 109-114.

RHODES, 1. 1968. The growth and development of some grass species under competitive stress. 1. Competition between seedlings, and between seedlings and established plants. Jl. Br. Grassld. Soc., 23, 129-136,

ROSS, M. ID. AND GREGORIUS, H.-R. 1983. Outcrossing and sex function in hermaphrodites: A resource-allocation model. Amer. Natur., 121, 204-222.

THOMSON, J. D. AND BARRETT, S. C. H. 1981. Temporal variation in gender in Aralia hispida Vent. (Araliaceae). Evolution, 35, 1094-1107.

WrimaRCK, A. 1968. Self-incompatibility in the Graminae. Hereditas, 60, 157-166. 